Pure and Applied Mathematics Quarterly

Volume 2, Number 3

(Special Issue: In honor of

Robert MacPherson, Part 1 of 3)

$817-836,2006$

\title{
Dimensions of Newton Strata in the Adjoint Quotient of Reductive Groups
}

\author{
Robert E. Kottwitz \\ Dedicated to R. MacPherson on his 60th birthday
}

\section{INTRODUCTION}

In [Cha00] Chai made a conjecture on the codimensions of Newton strata in Shimura varieties, which then led Rapoport [Rap05] to his conjecture on dimensions of affine Deligne-Lusztig varieties inside affine Grassmannians. The main goal of this paper is to show that exactly the same codimensions arise in a simpler context, that of the Newton stratification in the adjoint quotient of a reductive group. Along the way we study this stratification and then introduce the notion of defect, which we use to rewrite the codimension formula without having to use the greatest integer function.

The influence of the work of Chai and Rapoport on this paper is obvious. Less obvious is the influence of some joint work (as yet unpublished) with M. Goresky and R. MacPherson on codimensions of root-valuation strata in Lie algebras over Laurent power series fields. It is an especially great pleasure to acknowledge the influence of Goresky and MacPherson in a paper like this one, dedicated to Bob MacPherson on his 60th birthday. In addition I would like to thank T. Haines and M. Sabitova for some very helpful comments on an earlier version of this paper.

1.1. Preliminaries concerning the discretely valued field $F$. Let $\mathcal{O}$ be a complete discrete valuation ring with uniformizing element $\pi$ and algebraically closed residue field $k:=\mathcal{O} / \pi \mathcal{O}$. In this paper it turns out to be more convenient

Received October 17, 2005.

1991 Mathematics Subject Classification.Primary 11G18; Secondary 14G35, 14L05, 20G25.

Partially supported by NSF Grant DMS-0245639. 
to use the negative of the usual valuation. Thus our valuation on the fraction field $F$ of $\mathcal{O}$ is a surjective homomorphism

$$
\text { val : } F^{\times} \rightarrow \mathbb{Z}
$$

normalized so that $\operatorname{val}(\pi)=-1$. It is then appropriate to put $\operatorname{val}(0)=-\infty$. With these conventions the ultrametric inequality becomes

$$
\operatorname{val}(a+b) \leq \max \{\operatorname{val}(a), \operatorname{val}(b)\}
$$

with equality when the valuations of $a$ and $b$ are distinct.

Let $\bar{F}$ be an algebraic closure of $F$ and extend the given valuation on $F$ to a valuation on $\bar{F}$. Thus we now have a surjective homomorphism val $: \bar{F}^{\times} \rightarrow \mathbb{Q}$.

1.2. Classical Newton polygons. Let $f(T)=T^{n}+a_{1} T^{n-1}+\cdots+a_{n-1} T+a_{n}$ be a monic polynomial of degree $n$ with coefficients in $F$ and non-zero constant term $a_{n}$. The classical theory of Newton polygons (see [Rob80] for example) concerns the relation between the valuations of the $n$ roots of $f(T)$ in $\bar{F}$ and the valuations of the coefficients of $f(T)$. Recall that the valuations of the roots of $f$ are determined by the valuations of the coefficients of $f$ (but not the other way around). We now review the theory in greater detail.

Since $a_{n} \neq 0$, all $n$ roots of $f(T)$ are non-zero, and their valuations $\nu_{1}, \ldots, \nu_{n}$, when arranged in decreasing order $\nu_{1} \geq \cdots \geq \nu_{n}$, give us a well-defined point $\nu=\left(\nu_{1}, \ldots, \nu_{n}\right) \in \mathbb{Q}^{n}$.

We get another $n$-tuple $d=\left(d_{1}, \ldots, d_{n}\right)$ by putting $d_{i}=\operatorname{val}\left(a_{i}\right)$. Thus $d$ lies in $\tilde{\mathbb{Z}}^{n-1} \times \mathbb{Z}$, where $\tilde{\mathbb{Z}}:=\mathbb{Z} \cup\{-\infty\}$. Up to a sign, $a_{i}$ is the $i$-th elementary symmetric function of the roots, and it is clear that the valuation of each monomial contributing to $a_{i}$ has valuation $\leq \nu_{1}+\nu_{2}+\cdots+\nu_{i}$ with equality occurring for at least one monomial. Therefore

$$
d_{i} \leq \nu_{1}+\cdots+\nu_{i} \quad \text { for } i=1, \ldots, n-1
$$

and

$$
d_{i}=\nu_{1}+\cdots+\nu_{i} \quad \text { for } i=n .
$$

Moreover, when $\nu_{i}>\nu_{i+1}$, exactly one monomial has valuation $\nu_{1}+\cdots+\nu_{i}$, the rest having strictly smaller valuations, so that equality holds in (1.2.1) in this case.

Thus, as mentioned before, $\nu$ does not determine $d$. However, $d$ does determine $\nu$. For this we need the following partial order on $\mathbb{Q}^{n}:$ for $\mu, \mu^{\prime} \in \mathbb{Q}^{n}$ we write $\mu^{\prime} \leq \mu$ if and only if

$$
\mu_{1}^{\prime}+\cdots+\mu_{i}^{\prime} \leq \mu_{1}+\cdots+\mu_{i} \quad \text { for } i=1, \ldots, n-1
$$

and

$$
\mu_{1}^{\prime}+\cdots+\mu_{i}^{\prime}=\mu_{1}+\cdots+\mu_{i} \quad \text { for } i=n .
$$


It then turns out that $\nu$ is the unique smallest element in the set of all $\mu \in \mathbb{Q}^{n}$ satisfying the three conditions

$$
\begin{gathered}
\mu_{1} \geq \cdots \geq \mu_{n} \\
d_{i} \leq \mu_{1}+\cdots+\mu_{i} \quad \text { for } i=1, \ldots, n-1 \\
d_{i}=\mu_{1}+\cdots+\mu_{i} \quad \text { for } i=n .
\end{gathered}
$$

In classical language $\nu_{1}, \ldots, \nu_{n}$ are the slopes of the Newton polygon obtained from the $n$-tuple $d$.

This story is related to the reductive group $G L_{n}$ in two ways. First, an element $g \in G L_{n}(F)$ has a characteristic polynomial $f(T)$, whose roots are the $n$ eigenvalues of $g$. This provides a natural source of the kind of polynomials we have been considering. Second, as is well-known, the partial order $\leq$ and inequalities such as $\nu_{1} \geq \cdots \geq \nu_{n}$ and $\nu_{i}>\nu_{i+1}$ occur most naturally in the context of the root system for $G L_{n}$. Our first goal in this paper is to extend the theory of Newton polygons to other reductive groups, as has already been done for isocrystals with $G$-structure [Kot85],[RR96],[Cha00]. This requires some preparation involving root systems and reductive groups, all of which is standard, but which we review for the sake of completeness.

1.3. Root theoretic preliminaries. Consider a split connected reductive group $G$ over $\mathcal{O}$. We fix a split maximal torus $A$ in $G$ as well as a Borel subgroup $B=A U$ containing $A$, with $U$ denoting the unipotent radical of $B$. We assume that $B, A, U$ are defined over $\mathcal{O}$, but most of the time we will really be thinking about $G, A, B, U$ as algebraic groups over $F$. We also assume that the derived group of $G$ is simply connected. We denote this derived group by $G_{\mathrm{sc}}$. Thus there is an exact sequence

$$
1 \rightarrow G_{\mathrm{sc}} \rightarrow G \rightarrow D \rightarrow 1,
$$

where $D:=G / G_{\mathrm{sc}}$, a split torus. We write $A_{\mathrm{sc}}$ for the intersection of $A$ with $G_{\mathrm{sc}}$. Thus $A_{\mathrm{sc}}$ is a split maximal torus in $G_{\mathrm{sc}}$, and there is an exact sequence

$$
1 \rightarrow A_{\mathrm{sc}} \rightarrow A \rightarrow D \rightarrow 1,
$$

giving rise to the exact sequence

$$
0 \rightarrow X^{*}(D) \rightarrow X^{*}(A) \rightarrow X^{*}\left(A_{\mathrm{sc}}\right) \rightarrow 0
$$

of character groups.

Let $\alpha_{1}, \ldots, \alpha_{l} \in X^{*}(A)$ denote the simple roots of $A$, and let $\alpha_{1}^{\vee}, \ldots, \alpha_{l}^{\vee} \in$ $X_{*}\left(A_{\mathrm{sc}}\right)$ denote the corresponding simple coroots. We write $\Delta$ for the set of simple roots. The simple coroots form a $\mathbb{Z}$-basis for $X_{*}\left(A_{\mathrm{sc}}\right)$, and we write $\varpi_{1}, \ldots, \varpi_{l}$ for the corresponding dual basis for $X^{*}\left(A_{\mathrm{sc}}\right)$. Thus $\varpi_{1}, \ldots, \varpi_{l} \in X^{*}\left(A_{\mathrm{sc}}\right)$ are the fundamental weights for our root system. We now extend each fundamental 
weight $\varpi_{i}$ to a character $\omega_{i}$ on $A$. (This involves a choice, since such an extension is well-defined only modulo elements in $X^{*}(D)$.) Additionally, we choose a basis $\omega_{l+1}, \ldots, \omega_{n}$ for $X^{*}(D)$, where $n$ denotes the dimension of $A$. The characters $\omega_{1}, \ldots, \omega_{n}$ then form a $\mathbb{Z}$-basis for $X^{*}(A)$.

As an example, consider the case of $G L_{n}$ with the standard choice for $B=A U$. We identify both $X_{*}(A)$ and $X^{*}(A)$ with $\mathbb{Z}^{n}$. We also order the simple roots $\alpha_{1}, \ldots, \alpha_{n-1}$ in the usual way, so that $\left\langle\alpha_{i}, \nu\right\rangle=\nu_{i}-\nu_{i+1}$ for all $\nu \in X_{*}(A)$. Then for $i=1, \ldots, n$ we may take $\omega_{i}$ to be the weight $1^{i} 0^{n-i} \in \mathbb{Z}^{n}=X^{*}(A)$, the notation $1^{i} 0^{n-i}$ meaning that 1 is repeated $i$ times and that 0 is repeated $n-i$ times. Notice that for $\nu \in X_{*}(A)$ the expression $\nu_{1}+\cdots+\nu_{i}$ occurring earlier is equal to $\left\langle\omega_{i}, \nu\right\rangle$.

We will need the standard parabolic subgroups $P=M N$ of $G$, standard meaning that $P$ contains $B$. Here $N$ is the unipotent radical of $P$, and we always assume that the Levi subgroup $M$ is the unique one containing $A$. The set $\Delta$ decomposes as the disjoint union of $\Delta_{M}$ and $\Delta_{N}$, where $\Delta_{M}$ is the set of simple roots of $M$ and $\Delta_{N}$ is the set of simple roots of $G$ occurring in the Lie algebra of $N$. We write $A_{M}$ for the identity component of the center of $M$. Thus $A_{M}$ is a subtorus of $A$.

We will need the real vector space $\mathfrak{a}:=X_{*}(A) \otimes_{\mathbb{Z}} \mathbb{R}$. Sometimes we will also need its $\mathbb{Q}$-subspace $\mathfrak{a}_{\mathbb{Q}}:=X_{*}(A) \otimes_{\mathbb{Z}} \mathbb{Q}$. We say that $x \in \mathfrak{a}$ is dominant if $\langle\alpha, x\rangle \geq 0$ for all $\alpha \in \Delta$, and we write $\mathfrak{a}_{\mathrm{dom}}$ for the set of dominant elements in $\mathfrak{a}$. We also need the usual partial order on $\mathfrak{a}$ : for $x, y \in \mathfrak{a}$ we say that $x \leq y$ if and only if $y-x$ is a non-negative linear combination of simple coroots. In the case of $G L_{n}$ an element $\nu \in \mathfrak{a}=\mathbb{R}^{n}$ is dominant if and only if $\nu_{1} \geq \cdots \geq \nu_{n}$, and the partial order is the same as the one discussed earlier (but for $\mathbb{R}^{n}$ instead of $\mathbb{Q}^{n}$ ). Later we will also need the analogous partial order on the dual space $\mathfrak{a}^{*}$ : for $x, y \in \mathfrak{a}^{*}$ we say that $x \leq y$ if and only if $y-x$ is a non-negative linear combination of simple roots.

We write $W$ for the Weyl group of $A$ in $G$ and choose a $W$-invariant Euclidean inner product $(x, y)$ on $\mathfrak{a}$, which we occasionally use to identify $\mathfrak{a}$ with its dual.

More generally we also need $\mathfrak{a}_{M}:=X_{*}\left(A_{M}\right) \otimes_{\mathbb{Z}} \mathbb{R}$. The obvious inclusion $X_{*}\left(A_{M}\right) \subset X_{*}(A)$ lets us view $\mathfrak{a}_{M}$ as a subspace of $\mathfrak{a}$. In fact $\mathfrak{a}_{M}=\{x \in$ $\left.\mathfrak{a}:\langle\alpha, x\rangle=0 \quad \forall \alpha \in \Delta_{M}\right\}$. The subspace $\mathfrak{a}_{M}$ of $\mathfrak{a}$ has a natural complement, namely the span of the simple coroots for $M$. Thus $\mathfrak{a}_{M}$ is in a natural way a direct summand of $\mathfrak{a}$, and we write $p_{M}: \mathfrak{a} \rightarrow \mathfrak{a}_{M}$ for the projection map. Write $W_{M}$ for the Weyl group of $A$ in $M$. Identifying $W_{M}$ with a subgroup of $W$, we can also describe $\mathfrak{a}_{M}$ as the set of fixed points of $W_{M}$ on $\mathfrak{a}$, and $p_{M}$ as the map sending $x$ to the average $\left|W_{M}\right|^{-1} \sum_{w \in W_{M}} w x$ of the points in the $W_{M}$-orbit of $x$. Using our inner product, we can also view $p_{M}$ as orthogonal projection on $\mathfrak{a}_{M}$. 
We write $\Lambda_{M}$ for the quotient of $X_{*}(A)$ by the coroot lattice for $M$. Since we have assumed that the derived group of $G$ is simply connected, the same is true for $M$, and therefore $\Lambda_{M}$ is a free abelian group, which we identify with the image of $X_{*}(A)$ under $p_{M}$, so that $\Lambda_{M}$ becomes a lattice in $\mathfrak{a}_{M}$.

Returning to the standard parabolic subgroup $P=M N$, we obtain an open cone $\mathfrak{a}_{P}^{+}$in $\mathfrak{a}_{M}$ by putting

$$
\mathfrak{a}_{P}^{+}:=\left\{x \in \mathfrak{a}_{M}:\langle\alpha, x\rangle>0 \quad \forall \alpha \in \Delta_{N}\right\} .
$$

We then define $\Lambda_{P}^{+}$to be the intersection (in $\mathfrak{a}_{M}$ ) of the lattice $\Lambda_{M}$ and the cone $\mathfrak{a}_{P}^{+}$. Recall that $\mathfrak{a}_{\text {dom }}$ is the disjoint union of the cones $\mathfrak{a}_{P}^{+}$as $P$ varies through all standard parabolic subgroups. Finally we define a discrete subset $\mathcal{N}_{G}$ of $\mathfrak{a}_{\text {dom }}$ by

$$
\mathcal{N}_{G}:=\coprod_{P} \Lambda_{P}^{+}
$$

where $P$ runs over all standard parabolic subgroups.

Let us work out the example of $G L_{n}$. To get a standard parabolic subgroup $P=M N$ we need a partition $n=n_{1}+\cdots+n_{r}$ of $n$. Given $\nu \in \mathfrak{a}=\mathbb{R}^{n}$, we refer to its first $n_{1}$ entries as the first batch, its next $n_{2}$ entries as the second batch, and so on. There are $r$ batches in all. Then $\mathfrak{a}_{M}$ is the subspace of $\mathbb{R}^{n}$ consisting of $n$-tuples such that all entries in the first batch are equal to each other, all entries in the second batch are equal to each other, and so on. The lattice $\Lambda_{M}$ consists of such vectors which satisfy the additional condition that the common value $\bar{\nu}_{j}$ of the entries in the $j$-th batch satisfies $n_{j} \bar{\nu}_{j} \in \mathbb{Z}$. Finally, $\Lambda_{P}^{+}$consists of vectors satisfying all these conditions as well as the additional condition that $\bar{\nu}_{1}>\bar{\nu}_{2}>\cdots>\bar{\nu}_{r}$. The reader should observe that $\mathcal{N}_{G}$ is precisely the set of vectors arising as the slopes of the Newton polygon of some monic polynomial of degree $n$.

1.4. The closest point map $r: \mathfrak{a} \rightarrow \mathfrak{a}_{\text {dom }}$. With these definitions out of the way, we now turn to a general root theoretic construction which produces Newton polygons in the special case of $G L_{n}$. In fact this construction is a standard one, one viewpoint being that it is the most elementary case of Langlands' combinatorial lemma, another being that it is the root theoretic fact needed for the Langlands classification. An excellent textbook reference is [Kna86]. The basic idea is as follows. Let $x \in \mathfrak{a}$. Then there is a unique point $y \in \mathfrak{a}_{\text {dom }}$ that is closest to $x$ (in the metric on $\mathfrak{a}$ obtained from our Euclidean inner product). In the case of $G L_{n}$ the map $x \mapsto y$ is the map needed to produce the Newton polygon from the data of the valuations of the coefficients of the polynomial $f(T)$. (Strictly speaking, this is only true when every coefficient is non-zero.)

The next proposition summarizes all we need to know about $x \mapsto y$. Much of what we need is in [Kna86]; the rest will be verified later (see 2.1). 


\section{Proposition 1.4.1.}

(1) For all $x \in \mathfrak{a}$ there exists a unique point $r(x) \in \mathfrak{a}_{\text {dom }}$ that is closest to $x$. The map $r$ is a continuous retraction of $\mathfrak{a}$ onto $\mathfrak{a}_{\mathrm{dom}}$.

(2) Let $y \in \mathfrak{a}_{\mathrm{dom}}$, and let $P=M N$ be the unique standard parabolic subgroup such that $y \in \mathfrak{a}_{P}^{+}$. Then

$$
r^{-1}(y)=\left\{x \in \mathfrak{a}: p_{M}(x)=y \text { and } x \leq y\right\} .
$$

(3) The point $r(x)$ can also be characterized as the unique minimal element in the set $\left\{\mu \in \mathfrak{a}_{\mathrm{dom}}: x \leq \mu\right\}$. In other words, $x \leq r(x)$, and if $\mu \in \mathfrak{a}_{\mathrm{dom}}$ and $x \leq \mu$, then $r(x) \leq \mu$.

(4) $r\left(\mathfrak{a}_{\mathbb{Q}}\right)=\mathfrak{a}_{\mathbb{Q}} \cap \mathfrak{a}_{\mathrm{dom}}$.

(5) $r\left(X_{*}(A)\right)=\mathcal{N}_{G}$.

We actually need a slight generalization, since we will sometimes be taking the valuation of 0 , which will yield $-\infty$. To handle this it is best to proceed a bit differently. We will now use the map $x \mapsto\left(\left\langle\omega_{1}, x\right\rangle, \ldots,\left\langle\omega_{n}, x\right\rangle\right)$ to identify $\mathfrak{a}$ with $\mathbb{R}^{n}$. Put $\tilde{\mathbb{R}}:=\mathbb{R} \cup\{-\infty\}$ with the obvious topology, for which a neighborhood base at $-\infty$ is provided by the intervals $[-\infty, a)$. Then, as we will check later (see 2.2) the map $r$ extends continuously to a map, still called $r$, from $\tilde{\mathbb{R}}^{l} \times \mathbb{R}^{n-l}$ to $\mathfrak{a}_{\mathrm{dom}}$.

\section{Proposition 1.4.2.}

(1) The image under $r$ of $\tilde{\mathbb{Z}}^{l} \times \mathbb{Z}^{n-l}$ is $\mathcal{N}_{G}$.

(2) Let $\mu \in \mathfrak{a}_{\mathrm{dom}}$ and $d \in \tilde{\mathbb{R}}^{l} \times \mathbb{R}^{n-l}$. Then $r(d) \leq \mu$ if and only if $d_{i} \leq\left\langle\omega_{i}, \mu\right\rangle$ for $i=1, \ldots, l$ and $d_{i}=\left\langle\omega_{i}, \mu\right\rangle$ for $i=l+1, \ldots, n$.

(3) Let $\mu \in \mathfrak{a}_{\mathrm{dom}}$ and $d \in \tilde{\mathbb{R}}^{l} \times \mathbb{R}^{n-l}$. Then $r(d)=\mu$ if and only if

$$
\begin{aligned}
d_{i} & \leq\left\langle\omega_{i}, \mu\right\rangle \text { for all } i \in I_{\mu}, \text { and } \\
d_{i} & =\left\langle\omega_{i}, \mu\right\rangle \text { for all } i \notin I_{\mu},
\end{aligned}
$$

where $I_{\mu}:=\left\{i \in\{1, \ldots, l\}:\left\langle\alpha_{i}, \mu\right\rangle=0\right\}$.

This will be proved in subsection 2.3 .

1.5. Newton strata for the adjoint quotient $\mathbb{A}=A / W$. We will be interested in the quotient variety $A / W$. The ring $R$ of regular functions on $A / W$ is the $F$-algebra obtained as the subring of $W$-invariant elements in the group algebra $F\left[X^{*}(A)\right]$ of $X^{*}(A)$. When we view $\lambda \in X^{*}(A)$ as an element of $F\left[X^{*}(A)\right]$ we denote it by $e^{\lambda}$. For $i=1, \ldots, n$ we put $c_{i}:=\sum_{\lambda \in W \omega_{i}} e^{\lambda}$ (with $W \omega_{i}$ denoting the Weyl group orbit of $\omega_{i}$ in $\left.X^{*}(A)\right)$. For $i=l+1, \ldots, n$ we then have simply $c_{i}=e^{\omega_{i}}$. It is well-known (see [Bou02]) that the monomials $c_{1}^{d_{1}} \ldots c_{n}^{d_{n}}$ with $d_{i} \in \mathbb{Z}$ for all $i$ and $d_{i} \geq 0$ for $i=1, \ldots, l$ form a basis for the $F$-vector space $R$. In other 
words $R$ is the ring $F\left[c_{1}, \ldots, c_{n}, c_{l+1}^{-1}, \ldots, c_{n}^{-1}\right]$ obtained from the polynomial ring $F\left[c_{1}, \ldots, c_{n}\right]$ by inverting $c_{l+1}, \ldots, c_{n}$, and the morphism

$$
c: A \rightarrow \mathbb{A}^{l} \times \mathbb{G}_{m}^{n-l}
$$

defined by $c(a)=\left(c_{1}(a), \ldots, c_{n}(a)\right)$ identifies $A / W$ with $\mathbb{A}^{l} \times \mathbb{G}_{m}^{n-l}$. From now on we will write $\mathbb{A}$ for $A / W$, even though it is only an affine space when $G=G_{\text {sc }}$.

As an example, consider the case of $G L_{n}$, again taking $\omega_{i}$ to be the weight $1^{i} 0^{n-i} \in \mathbb{Z}^{n}=X^{*}(A)$. Writing $\left(a_{1}, \ldots, a_{n}\right)$ for the $n$ diagonal entries of $a \in A(\bar{F})$, we then have

$$
c_{i}(a)=\sum_{I} \prod_{j \in I} a_{j}
$$

in which the sum is taken over all subsets $I$ of $\{1, \ldots, n\}$ having cardinality $i$. In other words $c_{i}$ is the usual $i$-th elementary symmetric function of $a_{1}, \ldots, a_{n}$, so that the characteristic polynomial of $a$ is $T^{n}-c_{1} T^{n-1}+\cdots+(-1)^{n} c_{n}$. On the other hand the eigenvalues $a_{1}, \ldots, a_{n}$ of $a$ are obtained by evaluating certain characters of $A$ on $a$.

We now return to the general case. Let $a \in A(\bar{F})$. The example of $G L_{n}$ suggests that our goal should be to relate the valuations of the elements $c_{i}(a)$ to those of $\lambda(a)$ (for characters $\lambda \in X^{*}(A)$ ). To keep track of the numbers val $\lambda(a)$ we define an element $\nu_{a} \in \mathfrak{a}_{\mathbb{Q}}$ by requiring that

$$
\left\langle\lambda, \nu_{a}\right\rangle=\operatorname{val} \lambda(a) \quad \forall \lambda \in X^{*}(A) .
$$

Thus $a \mapsto \nu_{a}$ is a surjective homomorphism from $A(\bar{F})$ to $\mathfrak{a}_{\mathbb{Q}}$. Clearly $\nu_{w a}=w \nu_{a}$ for any $w \in W$.

Let $c=\left(c_{1}, \ldots, c_{n}\right) \in \bar{F}^{l} \times\left(\bar{F}^{\times}\right)^{n-l}=\mathbb{A}(\bar{F})$. Put $\tilde{\mathbb{Q}}:=\mathbb{Q} \cup\{-\infty\}$ and define an $n$-tuple $d_{c} \in \tilde{\mathbb{Q}}^{l} \times \mathbb{Q}^{n-l}$ by

$$
d_{c}:=\left(\operatorname{val} c_{1}, \ldots, \operatorname{val} c_{n}\right) .
$$

The next result, which will be proved in section 3 , shows how to recover the $W$-orbit of $\nu_{a}$ from $d_{c(a)}$.

Theorem 1.5.1. Let $a \in A(\bar{F})$. Then $r\left(d_{c(a)}\right)$ is the unique dominant element in the $W$-orbit of $\nu_{a}$.

We are more interested in $\mathbb{A}(F)$ than in $\mathbb{A}(\bar{F})$, and for $\mu \in \mathcal{N}_{G}$ we now put

$$
\begin{aligned}
\mathbb{A}(F)_{\mu} & :=\left\{c \in \mathbb{A}(F): r\left(d_{c}\right)=\mu\right\} \\
\mathbb{A}(F)_{\leq \mu}: & =\left\{c \in \mathbb{A}(F): r\left(d_{c}\right) \leq \mu\right\} .
\end{aligned}
$$

The sets $\mathbb{A}(F)_{\mu}$ are the Newton strata referred to in the title of this article. 
Theorem 1.5.2.

(1) The sets $\mathbb{A}(F)_{\mu}$ are non-empty and

$$
\mathbb{A}(F)=\coprod_{\mu \in \mathcal{N}_{G}} \mathbb{A}(F)_{\mu} .
$$

(2) $\mathbb{A}(F)_{\leq \mu}=\coprod_{\left\{\nu \in \mathcal{N}_{G}: \nu \leq \mu\right\}} \mathbb{A}(F)_{\nu}$.

(3) $\mathbb{A}(F)_{\leq \mu}$ consists of $n$-tuples $\left(c_{1}, \ldots, c_{n}\right) \in F^{l} \times\left(F^{\times}\right)^{n-l}=\mathbb{A}(F)$ such that

$$
\begin{array}{ll}
\operatorname{val} c_{i} \leq\left\langle\omega_{i}, \mu\right\rangle & \text { for } i=1, \ldots, l, \text { and } \\
\operatorname{val} c_{i}=\left\langle\omega_{i}, \mu\right\rangle & \text { for } i=l+1, \ldots, n .
\end{array}
$$

(4) $\mathbb{A}(F)_{\mu}$ consists of $n$-tuples $\left(c_{1}, \ldots, c_{n}\right) \in F^{l} \times\left(F^{\times}\right)^{n-l}=\mathbb{A}(F)$ such that

$$
\begin{array}{ll}
\operatorname{val} c_{i} \leq\left\langle\omega_{i}, \mu\right\rangle & \text { for all } i \in I_{\mu}, \text { and } \\
\operatorname{val} c_{i}=\left\langle\omega_{i}, \mu\right\rangle & \text { for all } i \notin I_{\mu},
\end{array}
$$

where $I_{\mu}:=\left\{i \in\{1, \ldots, l\}:\left\langle\alpha_{i}, \mu\right\rangle=0\right\}$.

(5) Let $c \in \mathbb{A}(F)$ and choose $a \in A(\bar{F})$ such that $c(a)=c$. Then $c \in \mathbb{A}(F)_{\mu}$ if and only if $\mu$ is the unique dominant element in the $W$-orbit of $\nu_{a}$.

Proof. (1) follows from the first part of Proposition 1.4.2, and (2) is clear from the definitions. (3) and (4) follow from the second and third parts of Proposition 1.4.2. Finally, (5) follows from Theorem 1.5.1.

1.6. Dimensions of Newton strata. Let us agree to assign dimensions (over $k)$ to fractional ideals $P^{j}:=\pi^{j} \mathcal{O}(j \in \mathbb{Z})$ as follows:

$$
\operatorname{dim} P^{j}=-j .
$$

This definition is reasonable since we then have

$$
\operatorname{dim} P^{i}-\operatorname{dim} P^{j}=j-i,
$$

and, when $j \geq i$, we can interpret $j-i$ as the $k$-dimension of $P^{i} / P^{j}$ if we use Greenberg's method [Gre61] to view $P^{i} / P^{j}$ as the set of $k$-points of a variety over $k$.

Similarly, we assign to the subset $P^{j_{1}} \times \cdots \times P^{j_{l}}$ of $F^{l}$ the dimension $-\left(j_{1}+\right.$ $\left.\cdots+j_{l}\right)$. Finally, for any coset $C$ of $\left(\mathcal{O}^{\times}\right)^{n-l}$ in $\left(F^{\times}\right)^{n-l}$, we assign to the subset $P^{j_{1}} \times \cdots \times P^{j_{l}} \times C$ of $F^{l} \times\left(F^{\times}\right)^{n-l}=\mathbb{A}(F)$ the dimension $-\left(j_{1}+\cdots+j_{l}\right)$. This is compatible with the heuristic idea that the codimension of $P^{j_{1}} \times \cdots \times P^{j_{l}} \times C$ in $P^{i_{1}} \times \cdots \times P^{i_{l}} \times C$ should be $\left(j_{1}+\cdots+j_{l}\right)-\left(i_{1}+\cdots+i_{l}\right)$ when $i_{\beta} \leq j_{\beta}$ for $\beta=1, \ldots, l$.

We see from the third part of Theorem 1.5.2 that the subset $\mathbb{A}(F)_{\leq \mu}$ of $\mathbb{A}(F)=$ $F^{l} \times\left(F^{\times}\right)^{n-l}$ is equal to

$$
P^{-\left[\left\langle\omega_{1}, \mu\right\rangle\right]} \times \cdots \times P^{-\left[\left\langle\omega_{l}, \mu\right\rangle\right]} \times C
$$


for a suitable coset $C$ of $\left(\mathcal{O}^{\times}\right)^{n-l}$ in $\left(F^{\times}\right)^{n-l}$. Here $[a]$ denotes the greatest integer in $a$ (for $a \in \mathbb{R}$ ). Therefore the dimension of $\mathbb{A}(F)_{\leq \mu}$ is given by

$$
\operatorname{dim} \mathbb{A}(F)_{\leq \mu}=\sum_{i=1}^{l}\left[\left\langle\omega_{i}, \mu\right\rangle\right] .
$$

These numbers are not particularly meaningful since they depend on the extensions $\omega_{i}$ of $\varpi_{i}$ we chose in 1.3. However, given $\mu, \nu \in \mathcal{N}_{G}$ with $\nu \leq \mu$, the set $\mathbb{A}(F)_{\leq \nu}$ is contained in $\mathbb{A}(F)_{\leq \mu}$ with $k$-codimension

$$
\sum_{i=1}^{l}\left[\left\langle\omega_{i}, \mu\right\rangle\right]-\sum_{i=1}^{l}\left[\left\langle\omega_{i}, \nu\right\rangle\right],
$$

a non-negative integer independent of the choice of extensions $\omega_{i}$. (To verify the independence statement use the fact that for any $\lambda \in X^{*}(D)$ and $\nu \in \mathcal{N}_{G}$ the number $\langle\lambda, \nu\rangle$ is an integer.)

1.7. Comparison with Chai's work. An expression like $\sum_{i=1}^{l}\left[\left\langle\omega_{i}, \mu\right\rangle\right]$ appears in Chai's [Cha00] conjectural formula for the dimensions of Newton strata in Shimura varieties and again in Rapoport's conjectural formula [Rap05] for the dimensions of affine Deligne-Lusztig varieties inside the affine Grassmannian. It is intriguing that it arises in such a simple way in the context of the Newton stratification on $\mathbb{A}(F)$.

Before comparing the codimensions (1.6.2) with those in Chai's article [Cha00] let us rewrite (1.6.2) slightly. We have extended the fundamental weights $\varpi_{i}$ to characters $\omega_{i} \in X^{*}(A)$. There is another way to extend the fundamental weights to "characters" on $A$, more precisely, to elements in $X^{*}(A)_{\mathbb{Q}}$. For this we use the direct sum decomposition $X^{*}(A)_{\mathbb{Q}}=X^{*}\left(A_{\mathrm{sc}}\right)_{\mathbb{Q}} \oplus X^{*}\left(A_{G}\right)_{\mathbb{Q}}$ to view the fundamental weights $\varpi_{i}$ as elements of $X^{*}(A)_{\mathbb{Q}}$ that happen to lie in the lattice $X^{*}\left(A_{\mathrm{sc}}\right)$ in the direct summand $X^{*}\left(A_{\mathrm{sc}}\right)_{\mathbb{Q}}$ of $X^{*}(A)_{\mathbb{Q}}$. In other words, for $i=1, \ldots, l$ we may view $\varpi_{i}$ as the unique element of $X^{*}(A)_{\mathbb{Q}}$ satisfying the conditions $\left\langle\varpi_{i}, \alpha_{j}^{\vee}\right\rangle=\delta_{i j}$ for $1 \leq j \leq l$ and $\left\langle\varpi_{i}, \nu\right\rangle=0$ for all $\nu \in \mathfrak{a}_{G}$.

Now consider a dominant coweight $\mu \in X_{*}(A)$ and an element $\nu \in \mathcal{N}_{G}$ such that $\nu \leq \mu$, so that in particular $\mu-\nu \in X_{*}\left(A_{\mathrm{sc}}\right)_{\mathbb{Q}}$. We are going to rewrite (1.6.2). Since $\mu \in X_{*}(A)$, the numbers $\left\langle\omega_{i}, \mu\right\rangle$ are integers, from which it follows that

$$
\left[\left\langle\omega_{i}, \mu\right\rangle\right]-\left[\left\langle\omega_{i}, \nu\right\rangle\right]=-\left[\left\langle\omega_{i}, \nu-\mu\right\rangle\right]=\left\lceil\left\langle\omega_{i}, \mu-\nu\right\rangle\right\rceil
$$

where $\lceil r\rceil$ denotes the smallest integer that is greater than or equal to the real number $r$. Moreover, since $\mu-\nu \in X_{*}\left(A_{\mathrm{sc}}\right)_{\mathbb{Q}}$, we have $\left\langle\omega_{i}, \mu-\nu\right\rangle=\left\langle\varpi_{i}, \mu-\nu\right\rangle$. We conclude that for dominant $\mu \in X_{*}(A)$ and $\nu \in \mathcal{N}_{G}$ such that $\nu \leq \mu$, the set 
$\mathbb{A}(F)_{\leq \nu}$ is contained in $\mathbb{A}(F)_{\leq \mu}$ with $k$-codimension

$$
\sum_{i=1}^{l}\left\lceil\left\langle\varpi_{i}, \mu-\nu\right\rangle\right\rceil .
$$

Comparing this with the last displayed formula in [Cha00, Thm. 7.4], one sees that the codimension of $\mathbb{A}(F)_{\leq \nu}$ in $\mathbb{A}(F)_{\leq \mu}$ coincides with the value predicted by Chai for the codimension of the Newton stratum indexed by $\nu$ in any Shimura variety with associated minuscule coweight $\mu$ (with $G$ split over $\mathbb{Q}_{p}$ ).

Rapoport's conjectural formula (see [Rap05], [Mie05, eqn. (0.2)]) for dimensions of affine Deligne-Lusztig varieties inside affine Grassmannians can be given a similar treatment. This has been done implicitly in [GHKR05], using the notion of defect that we will be discussing in the remainder of this introduction.

1.8. Definition of $d_{G}: \mathcal{N}_{G} \rightarrow \mathbb{Q}$. For a rational number $r$ let us write $\operatorname{fr}(r) \in$ $[0,1)$ for the fractional part of $r$; thus

$$
[r]=r-\operatorname{fr}(r) .
$$

One sees immediately that

$$
\sum_{i=1}^{l}\left[\left\langle\omega_{i}, \nu\right\rangle\right]=\left\langle\rho^{\prime}, \nu\right\rangle-\sum_{i=1}^{l} \operatorname{fr}\left\langle\omega_{i}, \nu\right\rangle,
$$

where $\rho^{\prime}:=\sum_{i=1}^{l} \omega_{i}$, an extension to $A$ of the usual character

$$
\rho:=\sum_{i=1}^{l} \varpi_{i}=\frac{1}{2} \sum_{\alpha>0} \alpha \in X^{*}\left(A_{\mathrm{sc}}\right) .
$$

We remind the reader that for any $\lambda \in X^{*}(D)$ and $\nu \in \mathcal{N}_{G}$ the number $\langle\lambda, \nu\rangle$ is an integer. Therefore $\operatorname{fr}\left\langle\omega_{i}, \nu\right\rangle$ is independent of the choice of extension $\omega_{i}$ of $\varpi_{i}$, and, moreover, $\operatorname{fr}\left\langle\omega_{i}, \nu\right\rangle=0$ for $i=l+1, \ldots, n$. Thus we may define a function

$$
d_{G}: \mathcal{N}_{G} \rightarrow \mathbb{Q}
$$

independent of the choice of extensions $\omega_{i}$, by putting

$$
d_{G}(\nu):=\sum_{i=1}^{l} \operatorname{fr}\left\langle\omega_{i}, \nu\right\rangle=\sum_{i=1}^{n} \operatorname{fr}\left\langle\omega_{i}, \nu\right\rangle .
$$

Using this definition, we may rewrite our formula (1.6.1) as

$$
\operatorname{dim} \mathbb{A}(F)_{\leq \nu}=\left\langle\rho^{\prime}, \nu\right\rangle-d_{G}(\nu) .
$$


1.9. Computation of $d_{G}(\nu)$. We are going to calculate $d_{G}(\nu)$ for all $\nu \in \mathcal{N}_{G}$. Since $\Lambda_{G}^{+}=\Lambda_{G}$, the set $\mathcal{N}_{G}$ contains the lattice $\Lambda_{G}$, and it turns out that the main point is to calculate $d_{G}(\nu)$ when $\nu \in \Lambda_{G}$. Indeed the general case may be reduced to this special one by means of the next lemma.

Lemma 1.9.1. Let $\nu \in \mathcal{N}_{G}$, and let $P=M N$ be the unique standard parabolic subgroup such that $\nu \in \Lambda_{P}^{+}$. Then there is an equality

$$
d_{G}(\nu)=d_{M}(\nu)
$$

where on the right side $\nu$ is viewed as an element of $\Lambda_{M} \subset \mathcal{N}_{M}$ and $d_{M}: \mathcal{N}_{M} \rightarrow \mathbb{Q}$ is the analog for $M$ of $d_{G}$.

Proof. The derived group of $M$ is simply connected, and one sees immediately that the characters $\omega_{i}$ play the same role for $M$ as they do for $G$. Therefore the $n$ numbers $\operatorname{fr}\left\langle\omega_{i}, \nu\right\rangle$ we get for $M$ are the same as the $n$ numbers we get for $G$.

We are now reduced to computing $d_{G}(\nu)$ when $\nu \in \Lambda_{G} \subset \mathcal{N}_{G}$. For this we need to define, for each such $\nu$, a non-negative integer $\operatorname{def}_{G}(\nu)$ called the defect of $\nu$. Recall that the extended affine Weyl group $\tilde{W}$ is the semidirect product $\tilde{W}=X_{*}(A) \rtimes W$, and therefore comes with a surjective homomorphism

$$
p: \tilde{W} \rightarrow W .
$$

Since $W$ acts trivially on the quotient $\Lambda_{G}$ of $X_{*}(A)$, there is also an obvious surjective homomorphism

$$
q: \tilde{W} \rightarrow \Lambda_{G},
$$

which is trivial on $W$ and on $X_{*}(A)$ is the canonical surjection $X_{*}(A) \rightarrow \Lambda_{G}$. The kernel of $q$ is the affine Weyl group. Inside $\tilde{W}$ we have the subgroup consisting of all elements that preserve the set of simple affine roots (equivalently, that preserve the base alcove in $\mathfrak{a}$ ), and $q$ restricts to an isomorphism from this subgroup onto $\Lambda_{G}$; inverting this isomorphism we obtain the usual section $s$ of $q$, a homomorphism

$$
s: \Lambda_{G} \rightarrow \tilde{W}
$$

such that $q s=\mathrm{id}$. The composition $p s$ is a homomorphism

$$
\text { ps }: \Lambda_{G} \rightarrow W \text {. }
$$

The defect $\operatorname{def}_{G}(\nu)$ is then defined to be

$$
\operatorname{def}_{G}(\nu):=\operatorname{dim} \mathfrak{a}-\operatorname{dim} \mathfrak{a}^{w_{\nu}},
$$

where $w_{\nu}:=p s(\nu)$. Here, for $w \in W$ we are using $\mathfrak{a}^{w}$ to denote the set of fixed points of $w$ in $\mathfrak{a}$. It may be worth noting that the difference $\operatorname{dim} \mathfrak{a}-\operatorname{dim} \mathfrak{a}^{w}$ also occurs in the dimension formula for affine Springer fibers (see [KL88] and [Bez96]). 
Theorem 1.9.2. For all $\nu \in \Lambda_{G} \subset \mathcal{N}_{G}$ there is an equality

$$
d_{G}(\nu)=\frac{1}{2} \operatorname{def}_{G}(\nu)
$$

In particular $d_{G}(\nu) \in \frac{1}{2} \mathbb{Z}$.

Proof. This will be proved in subsection 4.2.

1.10. $B(G)$ and $\operatorname{def}_{G}(b)$. The set $\mathcal{N}_{G}$ also arises in the closely related context of isocrystals with $G$-structure. As in [Kot85, RZ96, Kot97], given a split group $G$ over $\mathbb{Q}_{p}$ and a basic element $b \in G(L)$ (where $L$ is the completion of a maximal unramified extension of $\mathbb{Q}_{p}$ ), we denote by $J_{b}$ the inner form of $G$ obtained by twisting the action of the Frobenius automorphism $\sigma$ of $L / \mathbb{Q}_{p}$ on $G(L)$ by $b$, so that

$$
J_{b}\left(\mathbb{Q}_{p}\right)=\left\{g \in G(L): g^{-1} b \sigma(g)=b\right\} .
$$

For any connected reductive group $H$ over $\mathbb{Q}_{p}$ we denote by $\operatorname{rk}_{\mathbb{Q}_{p}}(H)$ the dimension of any maximal $\mathbb{Q}_{p}$-split torus of $H$. Recall from [Kot85] that there is a bijection $b \mapsto \nu_{b}$ from the set of $\sigma$-conjugacy classes of basic elements $b \in G(L)$ to the abelian group $\Lambda_{G}$.

Theorem 1.10.1. Let $\nu \in \Lambda_{G} \subset \mathcal{N}_{G}$ and let $b$ be any basic element in $G(L)$ such that $\nu_{b}=\nu$. Then $\operatorname{rk}_{\mathbb{Q}_{p}}(G)=\operatorname{dim} \mathfrak{a}$ and $\operatorname{rk}_{\mathbb{Q}_{p}}\left(J_{b}\right)=\operatorname{dim} \mathfrak{a}^{w_{\nu}}$, so that

$$
\operatorname{def}_{G}(\nu)=\operatorname{rk}_{\mathbb{Q}_{p}}(G)-\operatorname{rk}_{\mathbb{Q}_{p}}\left(J_{b}\right) \text {. }
$$

Proof. This will be proved in subsection 4.3.

More generally (see [Kot85, RR96]), provided that the derived group of $G$ is simply connected, as we once again assume, there is a canonical map $b \mapsto$ $\nu_{b} \in \mathcal{N}_{G}$ which induces a bijection from $B(G)$ (the set of $\sigma$-conjugacy classes in $G(L)$ ) to the set $\mathcal{N}_{G}$. For $b \in G(L)$ one can still define a group $J_{b}$ (again see [Kot85, RZ96, Kot97]) satisfying (1.10.1). If $\nu_{b} \in \Lambda_{P}^{+} \subset \mathcal{N}_{G}$ for standard $P=$ $M N$, then $b$ is $\sigma$-conjugate to a basic element $b_{M} \in M(L)$ such that $\nu_{b_{M}}=\nu_{b}$. Moreover, $J_{b}$ is isomorphic to $J_{b_{M}}$, an inner form of $M$. Combining Lemma 1.9.1 and Theorems 1.9.2 and 1.10.1, and noting that $G$ has the same $\mathbb{Q}_{p}$-rank as $M$, we obtain the

Corollary 1.10.2. For any $b \in G(L)$ there is an equality

$$
d_{G}\left(\nu_{b}\right)=\frac{1}{2} \operatorname{def}_{G}(b)
$$

where $\operatorname{def}_{G}(b)$ is defined by

$$
\operatorname{def}_{G}(b):=\operatorname{rk}_{\mathbb{Q}_{p}} G-\operatorname{rk}_{\mathbb{Q}_{p}} J_{b}
$$


This corollary was used implicitly in [GHKR05] in order to reformulate the dimension conjecture of Rapoport in terms of $\operatorname{def}_{G}(b)$.

1.11. What if the derived group of $G$ is not simply connected? The best way to proceed in this case is probably to choose a central extension $G^{\prime}$ of $G$ by a split torus, doing this in such a way that the derived group of $G^{\prime}$ is simply connected. For example one would study $P G L_{n}$ by relating it to $G L_{n}$.

\section{Proofs of Propositions 1.4.1 And 1.4.2}

2.1. Proof of Proposition 1.4.1. (1) For any non-empty closed convex subset $C$ in the Euclidean space $\mathfrak{a}$ and any $x \in \mathfrak{a}$, there exists a unique point $r(x) \in C$ closest to $x$. Obviously $r: \mathfrak{a} \rightarrow C$ is a retraction, and $r$ is continuous because it satisfies the inequality

$$
d\left(r\left(x_{1}\right), r\left(x_{2}\right)\right) \leq d\left(x_{1}, x_{2}\right),
$$

where $d(-,-)$ denotes the Euclidean metric on $\mathfrak{a}$. To prove this inequality we may replace $C$ by the line segment joining $r\left(x_{1}\right)$ and $r\left(x_{2}\right)$, and then the proof is easy.

(2) This is simply a reformulation of Lemma 8.56 in [Kna86].

(3) Uniqueness is clear since the partial order $\leq$ has the property that $x \leq x^{\prime}$ and $x^{\prime} \leq x$ imply $x=x^{\prime}$. We see from (2) that $x \leq r(x)$. It remains to prove that if $\mu \in \mathfrak{a}_{\mathrm{dom}}$ and $x \leq \mu$, then $r(x) \leq \mu$. Let $P=M N$ be the unique standard parabolic subgroup such that $r(x) \in \mathfrak{a}_{P}^{+}$. We claim that

$$
r(x)=p_{M}(x) \leq p_{M}(\mu) \leq \mu .
$$

Indeed, $r(x)=p_{M}(x)$ follows from (2). The inequality $p_{M}(x) \leq p_{M}(\mu)$ follows from the inequality $x \leq \mu$ since $p_{M}$ preserves $\leq$. Finally, the inequality $p_{M}(\mu) \leq \mu$ is a consequence of the dominance of $\mu$ (since $p_{M}(\mu)$ is the average of the points $w \mu\left(w \in W_{M}\right)$ and the dominance of $\mu$ implies that $\left.w \mu \leq \mu\right)$.

(4) The map $r$ carries $\mathfrak{a}_{\mathbb{Q}}$ into $\mathfrak{a}_{\mathbb{Q}} \cap \mathfrak{a}_{\text {dom }}$ since it follows from (2) that there exists standard $P=M N$ such that $r(x)=p_{M}(x)$. Since $r$ is a retraction, it maps $\mathfrak{a}_{\mathbb{Q}}$ onto $\mathfrak{a}_{\mathbb{Q}} \cap \mathfrak{a}_{\mathrm{dom}}$.

(5) Let $y \in \mathfrak{a}_{\mathrm{dom}}$ and suppose that $y \in \mathfrak{a}_{P}^{+}$for standard $P=M N$. Then, using (2), we see that $y \in r\left(X_{*}(A)\right) \Longleftrightarrow p_{M}^{-1}(y) \cap\{x \in \mathfrak{a}: x \leq y\}$ meets $X_{*}(A)$. This happens $\Longleftrightarrow p_{M}^{-1}(y)$ meets $X_{*}(A) \Longleftrightarrow y \in \mathcal{N}_{G}$.

2.2. Continuity of $r: \tilde{\mathbb{R}}^{l} \times \mathbb{R}^{n-l} \rightarrow \mathfrak{a}_{\mathrm{dom}}$. The reader might find it helpful to draw a picture for $G=S L_{3}$, in which case it is clear that $r: \mathbb{R}^{n} \rightarrow \mathfrak{a}_{\text {dom }}$ extends continuously to $\tilde{\mathbb{R}}^{l} \times \mathbb{R}^{n-l} \rightarrow \mathfrak{a}_{\mathrm{dom}}$. 
Before handling the general case, we pause to transcribe part (2) of Proposition 1.4.1 using our identification $\mathfrak{a}=\mathbb{R}^{n}$. The statement becomes: for standard $P=M N$ and $y \in \mathfrak{a}_{P}^{+}$, the fiber $r^{-1}(y)$ is the set of $d=\left(d_{1}, \ldots, d_{n}\right) \in \mathbb{R}^{n}$ such that

$$
\begin{aligned}
& d_{i} \leq\left\langle\omega_{i}, y\right\rangle \text { for all } i \in I_{M}, \text { and } \\
& d_{i}=\left\langle\omega_{i}, y\right\rangle \text { for all } i \notin I_{M},
\end{aligned}
$$

where $I_{M}:=\left\{i \in\{1, \ldots, l\}: \alpha_{i} \in \Delta_{M}\right\}$.

Now we prove that $r$ extends continuously. The proof will use a map $d \mapsto d^{\prime}$ from $\mathbb{R}^{n}$ to itself that we will now define. We begin with the special case in which $G=G_{\mathrm{sc}}$, so that $n=l$ and $\omega_{i}=\varpi_{i}(1 \leq i \leq l)$. It is now convenient to use our $W$-invariant inner product to identify $\mathfrak{a}^{*}$ with $\mathfrak{a}$, so that $\omega_{i}$ can be regarded as an element of $\mathfrak{a}$. Since any $y \in \mathfrak{a}_{P}^{+}$is a strictly positive linear combination of $\omega_{i}$ $\left(i \notin I_{M}\right)$, the numbers $\left\langle\omega_{i}, y\right\rangle$ occurring in (2.2.1) are non-negative for all $i$ and strictly positive when $i \notin I_{M}$, and therefore we see that $r(d)=y \Longleftrightarrow r\left(d^{\prime}\right)=y$, where $d^{\prime}=\left(d_{1}^{\prime}, \ldots, d_{l}^{\prime}\right) \in \mathbb{R}^{l}$ is defined by

$$
d_{i}^{\prime}:=\max \left\{d_{i}, 0\right\}
$$

In the general case we write $\mathfrak{a}=\mathbb{R}^{n}$ as $\mathfrak{a}_{\mathrm{sc}} \oplus \mathfrak{a}_{G}$, where $\mathfrak{a}_{\mathrm{sc}}$ is the analog of $\mathfrak{a}$ for the group $G_{\mathrm{sc}}$. For $d=\left(d_{\mathrm{sc}}, z\right) \in \mathfrak{a}_{\mathrm{sc}} \oplus \mathfrak{a}_{G}$ we define $d^{\prime} \in \mathfrak{a}$ by $d^{\prime}:=\left(d_{\mathrm{sc}}^{\prime}, z\right)$, where $d_{\mathrm{sc}} \mapsto d_{\mathrm{sc}}^{\prime}$ is the map we just defined from $\mathfrak{a}_{\mathrm{sc}}=\mathbb{R}^{l}$ to itself. Again we have $r(d)=y \Longleftrightarrow r\left(d^{\prime}\right)=y$. In other words $r: \mathbb{R}^{n} \rightarrow \mathfrak{a}_{\mathrm{dom}}$ is the composition $r t$, where $t$ is the map $d \mapsto d^{\prime}$. It is clear that $t$ extends continuously to a map

$$
\tilde{\mathbb{R}}^{l} \times \mathbb{R}^{n-l} \rightarrow \mathbb{R}^{n} .
$$

Indeed, for this we may as well assume that $G=G_{\mathrm{sc}}$, in which case the desired extension of $t$ is still given by $d \mapsto d^{\prime}$, with $\max \left\{d_{i}, 0\right\}$ interpreted as 0 when $d_{i}=-\infty$. Therefore $r$ extends continuously.

2.3. Proof of Proposition 1.4.2. As a byproduct of the proof we just gave, we see that for our extended map $r: \tilde{\mathbb{R}}^{l} \times \mathbb{R}^{n-l} \rightarrow \mathfrak{a}_{\text {dom }}$ and $y \in \mathfrak{a}_{P}^{+}$, the fiber $r^{-1}(y)$ is given by the set of $d \in \tilde{\mathbb{R}}^{l} \times \mathbb{R}^{n-l}$ satisfying (2.2.1). This proves part (3) of Proposition 1.4.2, and it remains only to prove parts (1) and (2).

(1) We already know from Proposition 1.4.1 that $r\left(\mathbb{Z}^{n}\right)=\mathcal{N}_{G}$, so we just need to see that allowing some coordinates of $d \in \tilde{\mathbb{Z}}^{l} \times \mathbb{Z}^{n-l}$ to be $-\infty$ does not give anything new. This is clear from the discussion in subsection 2.2, since $r(d)$ does not change when each $-\infty$ occurring as a coordinate is replaced by some sufficiently negative integer.

(2) Put $y:=r(d)$. First we prove $(\Longrightarrow)$. So assume that $y \leq \mu$, which means that $\left\langle\omega_{i}, y\right\rangle \leq\left\langle\omega_{i}, \mu\right\rangle$ for $i=1, \ldots, l$ and $\left\langle\omega_{i}, y\right\rangle=\left\langle\omega_{i}, \mu\right\rangle$ for $i=l+1, \ldots, n$. 
This, together with (2.2.1) (which we know is true since we have already proved part (3) of this proposition), shows that

$$
\begin{aligned}
d_{i} & \leq\left\langle\omega_{i}, \mu\right\rangle \text { for all } i=1, \ldots, l, \text { and } \\
d_{i} & =\left\langle\omega_{i}, \mu\right\rangle \text { for all } i=l+1, \ldots, n,
\end{aligned}
$$

as desired.

Next we prove $(\Longleftarrow)$. Assume that $(2.3 .1)$ holds. Consider the point $d^{\prime} \in \mathbb{R}^{n}$ constructed from $d$ in subsection 2.2. Then (2.3.1) holds with $d$ replaced by $d^{\prime}$, or in other words, $d^{\prime}$ satisfies $d^{\prime} \leq \mu$. From part (3) of Proposition 1.4.1 we see that $r\left(d^{\prime}\right) \leq \mu$. Since $r(d)=r\left(d^{\prime}\right)$, this concludes the proof.

\section{Proof of Theorem 1.5.1}

Replacing $a$ by a suitable $W$-conjugate, we may assume that $\nu_{a}$ is dominant. We must then show that $r\left(d_{c(a)}\right)=\nu_{a}$. Let $P=M N$ be the unique standard parabolic subgroup such that $\nu_{a} \in \mathfrak{a}_{P}^{+}$. Then, appealing to part (3) of Proposition 1.4.2, we see that we must prove that

$$
\begin{aligned}
& \operatorname{val} c_{i}(a) \leq\left\langle\omega_{i}, \nu_{a}\right\rangle \text { for all } i \in I_{M}, \text { and } \\
& \operatorname{val} c_{i}(a)=\left\langle\omega_{i}, \nu_{a}\right\rangle \text { for all } i \notin I_{M},
\end{aligned}
$$

where $I_{M}:=\left\{i \in\{1, \ldots, l\}: \alpha_{i} \in \Delta_{M}\right\}$.

For $i=l+1, \ldots, n$ we have $c_{i}(a)=\omega_{i}(a)$, and therefore (3.0.3) holds for such $i$. Now suppose that $i$ is in the range $i=1, \ldots, l$. Then

$$
c_{i}(a)=\sum_{\lambda \in W \omega_{i}} \lambda(a)
$$

so that

$$
\operatorname{val} c_{i}(a) \leq \max \left\{\left\langle w \omega_{i}, \nu_{a}\right\rangle: w \in W\right\} .
$$

Since $\omega_{i}$ is dominant, $w \omega_{i} \leq \omega_{i}$, and this implies ( $\nu_{a}$ being dominant) that

$$
\left\langle w \omega_{i}, \nu_{a}\right\rangle \leq\left\langle\omega_{i}, \nu_{a}\right\rangle .
$$

This proves (3.0.2) and shows that in order to prove (3.0.3), it is enough to show that, if $i \notin I_{M}$, then

$$
\left\langle w \omega_{i}, \nu_{a}\right\rangle<\left\langle\omega_{i}, \nu_{a}\right\rangle
$$

unless $w \omega_{i}=\omega_{i}$.

We already noted that $w \omega_{i} \leq \omega_{i}$, which means that

$$
\omega_{i}-w \omega_{i}=\sum_{j=1}^{l} n_{j} \alpha_{j}
$$


for some non-negative integers $n_{1}, \ldots, n_{l}$. Therefore the difference between the two sides of (3.0.4) is

$$
\sum_{j=1}^{l} n_{j}\left\langle\alpha_{j}, \nu_{a}\right\rangle .
$$

Since $\left\langle\alpha_{j}, \nu_{a}\right\rangle \geq 0$, all terms in this sum are non-negative, and thus it will suffice to show that the $i$-th term is strictly positive. Since $i \notin I_{M}$, we do know that $\left\langle\alpha_{i}, \nu_{a}\right\rangle>0$, and it remains only to prove that $n_{i}>0$.

Recall our $W$-invariant inner product $(\cdot, \cdot)$ on $\mathfrak{a}$, which we now use to identify $\mathfrak{a}$ with its dual; each simple root $\alpha_{j}$ is then identified with a strictly positive multiple of the corresponding simple coroot $\alpha_{j}^{\vee}$. Taking the inner product of each side of (3.0.5) with $\omega_{i}$, we see that $n_{i}$ is strictly positive if and only if

$$
\left(\omega_{i}-w \omega_{i}, \omega_{i}\right)>0 .
$$

For any pair $v, v^{\prime}$ of distinct non-zero vectors having the same Euclidean norm, the inner product $\left(v-v^{\prime}, v\right)$ is strictly positive (Cauchy-Schwarz inequality). Applying this observation to the vectors $\omega_{i}, w \omega_{i}$ completes the proof.

\section{Proof of Theorems 1.9.2 And 1.10.1}

4.1. A lemma about the extended affine Weyl group $\tilde{W}$. Recall from before the surjective homomorphisms $p: \tilde{W} \rightarrow W, q: \tilde{W} \rightarrow \Lambda_{G}$, and the section $s: \Lambda_{G} \rightarrow \tilde{W}$ of $q$. Write $V$ for $\mathfrak{a}^{*} \otimes_{\mathbb{R}} \mathbb{C}$. We are interested in the reflection representation of $W$ on $V$, which we then regard as an $n$-dimensional representation of $\Lambda_{G}$ using the homomorphism $p s: \Lambda_{G} \rightarrow W$. The composition

$$
X_{*}\left(A_{G}\right) \hookrightarrow X_{*}(A) \rightarrow \Lambda_{G}
$$

identifies $X_{*}\left(A_{G}\right)$ with a subgroup of finite index in $\Lambda_{G}$, and this subgroup acts trivially on $V$; therefore $V$ must decompose as the direct sum of $n$ 1-dimensional representations of $\Lambda_{G}$. We are going to describe the $n$ characters of $\Lambda_{G}$ so obtained.

For each $i=1, \ldots, n$ we define a character $\chi_{i}: \Lambda_{G} \rightarrow \mathbb{C}^{\times}$as the following composition:

$$
\Lambda_{G} \hookrightarrow \mathfrak{a}_{G} \stackrel{\left\langle\omega_{i}, \cdot\right\rangle}{\longrightarrow} \mathbb{R} \rightarrow \mathbb{R} / \mathbb{Z} \stackrel{\exp (2 \pi i \cdot)}{\longrightarrow} \mathbb{C}^{\times} .
$$

Of course $\chi_{i}$ is trivial for $i=l+1, \ldots, n$, since $\left\langle\omega_{i}, \cdot\right\rangle$ then takes integral values on the lattice $\Lambda_{G}$ in $\mathfrak{a}_{G}$.

Lemma 4.1.1. As a $\Lambda_{G}$-module $V$ is isomorphic to the direct sum $\chi_{1} \oplus \cdots \oplus \chi_{n}$ of the 1-dimensional representations $\chi_{i}$. 
Proof. We already remarked that $V$ is really a representation of the quotient $\Lambda_{G} / X_{*}\left(A_{G}\right)$ of $\Lambda_{G}$. Moreover each character $\chi_{i}$ is clearly trivial on the subgroup $X_{*}\left(A_{G}\right)$. Therefore our problem really concerns the quotient $\Lambda_{G} / X_{*}\left(A_{G}\right)$, which may be identified with a subgroup of $P\left(R^{\vee}\right) / Q\left(R^{\vee}\right)$ (Bourbaki's notation), where $R^{\vee}$ is the coroot system of $G, P\left(R^{\vee}\right)$ is the lattice of weights for $R^{\vee}$, and $Q\left(R^{\vee}\right)=$ $X_{*}\left(A_{\mathrm{sc}}\right)$ is the coroot lattice for $G$.

How do we express $\chi_{i}(1 \leq i \leq l)$ in terms of $P\left(R^{\vee}\right) / Q\left(R^{\vee}\right)$ ? We use $\exp (2 \pi i \cdot)$ to identify $\mathbb{R} / \mathbb{Z}$ with the unit circle in $\mathbb{C}^{\times}$, and $\mathbb{Q} / \mathbb{Z}$ with the group of complex roots of unity. For $\nu \in \Lambda_{G} \subset \mathfrak{a}_{G}$ pick $\mu \in X_{*}(A)$ such that $p_{G}(\mu)=\nu$. We then claim that $\chi_{i}(\nu)$ is equal to $-\left\langle\varpi_{i}, \bar{\mu}\right\rangle \in \mathbb{Q} / \mathbb{Z} \hookrightarrow \mathbb{C}^{\times}$, where $\bar{\mu}$ denotes the image of $\mu$ under

$$
X_{*}(A) \rightarrow \Lambda_{G} \rightarrow \Lambda_{G} / X_{*}\left(A_{G}\right) \hookrightarrow P\left(R^{\vee}\right) / Q\left(R^{\vee}\right) .
$$

Indeed, since $\left\langle\omega_{i}, \mu\right\rangle \in \mathbb{Z}$, the rational numbers $\left\langle\omega_{i}, \nu\right\rangle$ and $\left\langle\omega_{i}, \nu-\mu\right\rangle$ become equal in $\mathbb{Q} / \mathbb{Z}$. Moreover, since $\nu-\mu \in X_{*}\left(A_{\text {sc }}\right)_{\mathbb{Q}}$, we have

$$
\left\langle\omega_{i}, \nu-\mu\right\rangle=\left\langle\varpi_{i}, \nu-\mu\right\rangle=-\left\langle\varpi_{i}, \mu\right\rangle .
$$

Thus our entire problem can be reformulated in terms of the adjoint group $G_{\text {ad }}$ of $G$. So for the rest of this proof we will consider an adjoint group $G$ and its extended affine Weyl group $\tilde{W}$. Thus $\Lambda_{G}$ is now the finite abelian group $\Lambda:=P\left(R^{\vee}\right) / Q\left(R^{\vee}\right)$ and $\chi_{i}(i=1, \ldots, l)$ has become the character $\chi_{i}: \Lambda \rightarrow$ $\mathbb{Q} / \mathbb{Z} \hookrightarrow \mathbb{C}^{\times}$defined by

$$
\chi_{i}(\bar{\nu})=-\left\langle\varpi_{i}, \nu\right\rangle,
$$

where $\nu \in P\left(R^{\vee}\right)$ is any representative for $\bar{\nu} \in \Lambda$.

When $G$ decomposes as a direct product, our problem decomposes accordingly, and therefore we may as well assume that $G$ is a simple adjoint group. At this point we use the classification of root systems. All the information we need can be obtained easily from the tables of root systems in Bourbaki [Bou02]. This is obviously the case for the numbers $\left\langle\varpi_{i}, \nu\right\rangle$, but what about the decomposition of the $\Lambda$-module $V$ as a sum of characters?

We can also consider the representation $V^{\prime}$ of $\tilde{W}$ on the space of affine linear (complex-valued) functions on $\mathfrak{a}$. The simple affine roots give a $\mathbb{C}$-basis for $V^{\prime}$, and $\Lambda$ permutes this basis. Thus the representation on $V^{\prime}$ of $\Lambda$ is the permutation representation obtained from its action on the set of vertices of the affine Dynkin diagram of $G$; this information too is contained in Bourbaki. We have the short exact sequence

$$
0 \rightarrow \mathbb{C} \hookrightarrow V^{\prime} \rightarrow V \rightarrow 0,
$$

with $\mathbb{C} \hookrightarrow V^{\prime}$ given by the constant functions on $\mathfrak{a}$. Therefore the assertion we need to check (for each root system) is that the set of $l+1$ characters of $\Lambda$ we get from its permutation representation on the vertices of the affine Dynkin diagram consists of the trivial character together with the $l$ characters $\chi_{i}$. 
For example in type $A_{n-1}$ the group $\Lambda$ is cyclic of order $n$ and permutes the $n$ vertices of the affine Dynkin diagram cyclically, so that each character of $\Lambda$ appears once in this $n$-dimensional permutation representation. In Bourbaki's numbering of the fundamental weights, $\chi_{1}$ generates the character group of $\Lambda$ and $\chi_{i}(i=1, \ldots, n-1)$ is the $i$-th power of $\chi_{1}$.

As another example, for $E_{6}$ the group $\Lambda$ is cyclic of order 3 , and has three orbits on the set of vertices of the affine Dynkin diagram, two orbits having three elements, and one having one element. Therefore $V^{\prime}$ decomposes as a sum of two copies of each of the non-trivial characters of $\Lambda$ and three copies of the trivial character. Again using Bourbaki's numbering system, we find that $\chi_{2}=\chi_{4}$ are trivial, $\chi_{1}=\chi_{5}$ are one of the two non-trivial characters, and $\chi_{3}=\chi_{6}$ are the other non-trivial character. So it works! One checks just as easily that it works for each simple root system.

We remark that since the reflection representation is defined over $\mathbb{Q}$, it was only a matter of convenience that we used $\exp (2 \pi i \cdot)$ to identify $\mathbb{Q} / \mathbb{Z}$ with the group of complex roots of unity. Any other identification of the two groups would have worked just as well.

4.2. Proof of Theorem 1.9.2. We will prove Theorem 1.9.2 by combining Lemma 4.1.1 with an argument of the same kind that Bezrukavnikov used to prove [Bez96, Lemma 3]. The characters $\chi_{i}(i=1, \ldots, n)$ introduced in subsection 4.1 take values in $\mathbb{Q} / \mathbb{Z} \hookrightarrow \mathbb{C}^{\times}$. Theorem 1.9 .2 involves the fractional parts of $\left\langle\omega_{i}, \nu\right\rangle$. We identify $\mathbb{Q} / \mathbb{Z}$ with the set of rational numbers in the interval $[0,1)$, so that $\chi_{i}$ now takes values in $[0,1)$. With this convention, we must show that for $\nu \in \Lambda_{G}$

$$
2 \sum_{i=1}^{n} \chi_{i}(\nu)=\operatorname{dim} \mathfrak{a}-\operatorname{dim} \mathfrak{a}^{w}
$$

where $w=w_{\nu}=p s(\nu)$. Since the reflection representation $V$ is self-contragredient, the multiplicity of $\chi_{i}$ in $V$ is the same as that of $\chi_{i}^{-1}$. Therefore the left side of (4.2.1) is equal to

$$
\sum_{i=1}^{n} \chi_{i}(\nu)+\chi_{i}^{-1}(\nu) .
$$

Now note that $\chi_{i}(\nu)+\chi_{i}^{-1}(\nu)$ is 1 if $\chi_{i}(\nu) \neq 0 \in \mathbb{Q} / \mathbb{Z}$ and is 0 otherwise. Therefore, using Lemma 4.1.1, we see that the left side of (4.2.1) is dim $\mathfrak{a}$ minus the number of times 1 occurs as an eigenvalue of $w$ acting on $\mathfrak{a}$, namely $\operatorname{dim} \mathfrak{a}^{w}$.

4.3. Proof of Theorem 1.10.1. In this theorem, we are given $\nu \in \Lambda_{G}$, and we are interested in basic elements $b \in G(L)$ such that $\nu_{b}=\nu$. There is a unique $\sigma$-conjugacy class of such elements, and since the isomorphism class of $J_{b}$ depends only on the $\sigma$-conjugacy class of $b$, it suffices to prove the theorem for 
any particular $b$ we like. The most convenient choice for $b$ is obtained as follows. The extended affine Weyl group $\tilde{W}$ can be identified with $N_{G}(A)\left(\mathbb{Q}_{p}\right) / A\left(\mathbb{Z}_{p}\right)$, where $N_{G}(A)$ denotes the normalizer of $A$ in $G$. We take as $b$ any element of $N_{G}(A)\left(\mathbb{Q}_{p}\right)$ that represents the element $\tilde{w} \in \tilde{W}$ obtained as the image of $\nu$ under our injection $s: \Lambda_{G} \rightarrow \tilde{W}$. [Since $\sigma(b)=b$ and $\tilde{w}$ has finite order modulo $A_{G}\left(\mathbb{Q}_{p}\right)$, the element $b$ is basic. Moreover it is clear that $\nu_{b}=\nu$.]

So we may work with this particular $b$, the advantage of which is that $\operatorname{Int}(b)$ (conjugation by $b$ ) preserves both $A$ and the standard Iwahori subgroup of $G$. Since $J_{b}$ is obtained from $G$ by twisting the Frobenius automorphism $\sigma$ by $\operatorname{Int}(b)$, the twist $A_{\tilde{w}}$ of $A$ by $\tilde{w}$ is an unramified maximal torus of $J_{b}$, whose split component is a maximal split torus in $J_{b}$ (by Bruhat-Tits theory). Therefore $\operatorname{rk}_{\mathbb{Q}_{p}}\left(J_{b}\right)$ is equal to the dimension of the split component of $A_{\tilde{w}}$. Since $\tilde{w}$ acts on $X_{*}(A)$ via $w_{\nu}=p(\tilde{w})$, we conclude that

$$
\operatorname{rk}_{\mathbb{Q}_{p}}\left(J_{b}\right)=\operatorname{dim} \mathfrak{a}^{w_{\nu}},
$$

as desired.

\section{REFERENCES}

[Bez96] R. Bezrukavnikov, The dimension of the fixed point set on affine flag manifolds, Math. Res. Lett. 3 (1996), 185-189.

[Bou02] N. Bourbaki, Lie groups and Lie algebras. Chapters 4-6, Elements of Mathematics (Berlin), Springer-Verlag, Berlin, 2002.

[Cha00] C.-L. Chai, Newton polygons as lattice points, Amer. J. Math. 122 (2000), no. 5, 967-990.

[GHKR05] U. Görtz, T. Haines, R. Kottwitz, and D. Reuman, Dimensions of some affine Deligne-Lusztig varieties, math.AG/0504443, 2005.

[Gre61] M. Greenberg, Schemata over local rings, Ann. of Math. (2) 73 (1961), 624-648.

[KL88] D. Kazhdan and G. Lusztig, Fixed point varieties on affine flag manifolds, Israel J. Math. 62 (1988), 129-168.

[Kna86] A. Knapp, Representation theory of semisimple groups, Princeton University Press, Princeton, N.J., 1986.

[Kot85] R. Kottwitz, Isocrystals with additional structure, Compositio Math. 56 (1985), 201220 .

[Kot97] R. Kottwitz, Isocrystals with additional structure. II, Compositio Math. 109 (1997), 255-339.

[Mie05] E. Mierendorff, Moduli spaces of p-divisible groups, math.AG/0502320, 2005.

[Rap05] M. Rapoport, A guide to the reduction modulo $p$ of Shimura varieties, Astérisque (2005), no. 298, 271-318.

[RR96] M. Rapoport and M. Richartz, On the classification and specialization of Fisocrystals with additional structure, Compositio Math. 103 (1996), 153-181.

[RZ96] M. Rapoport and Th. Zink, Period spaces for p-divisible groups, Annals of Mathematics Studies, vol. 141, Princeton University Press, Princeton, NJ, 1996.

[Rob80] P. Robba, Lemmes de Hensel pour les opérateurs différentiels. Application à la réduction formelle des équations différentielles, Enseign. Math. (2) 26 (1980), no. 34, 279-311 (1981). 
Robert E. Kottwitz

Department of Mathematics

University of Chicago

5734 University Avenue

Chicago, Illinois 60637

E-mail: kottwitz@math.uchicago.edu 